\title{
Prediction of Geraniol Bond Mode in Aspergillus niger Linalool
} Dehydratase - Isomerase

\author{
Yelfi Anwar1,2, Andrianopsyah Mas Jaya Putra ${ }^{2,3}$, Elvina Dhiaul Iftitah ${ }^{4,5}$, \\ Partomuan Simanjuntak $^{6,7}$, Shirly Kumala ${ }^{*}$ \\ ${ }^{1}$ Doctoral Program, Faculty of Pharmacy, University of Pancasila, Jakarta, Indonesia \\ 2 Faculty of Pharmacy, University of 17 August 1945, Jakarta, Indonesia \\ 3 Pusat Penelitian Kimia, Lembaga Ilmu Pengetahuan Indonesia \\ ${ }^{4}$ Essential Oil Institute, University of Brawijaya, Malang, Indonesia \\ 5 Department of Chemistry, Faculty of Math and Science, University of Brawijaya, Malang, Indonesia. \\ ${ }^{6}$ Pusat Penelitian Bioteknologi, Lembaga Ilmu Pengetahuan Indonesia. \\ ${ }^{7}$ Faculty of Pharmacy, University of Pancasila, Jakarta, Indonesia
}

\begin{abstract}
Geraniol is a very valuable aroma chemical and has wide use in fragrances and aroma compound. Geraniol biotransformation by Aspergillus niger has been studied. The main bioconversion products obtained from geraniol and liquid culture of $A$. niger are linalool and alpha-terpineol. Linalool plays a major role in anti-inflammatory, antibacterial and antioxidant activities. This study aims to know the interaction of geraniol in Aspergillus niger enzyme with docking molecular. Comparative modelling of Aspergillus niger enzyme was conducted by means of one of the crystal structures of Linalool Dehydratase - Idomerase (LDI) as a template. The best model of this comparative modelling was then used in docking molecular to investigate geraniol binding mode inactive site enzyme of Aspergillus niger. Inactive site enzyme of Aspergillus niger, geraniol is located with hydrophobic and hydrogen bonds: Amino acid - the amino acids are Asn 105, Arg 96, Lys 112 inactive site - OH with hydrogen bond, Arg 97 inactive site - CH3 with hydrophobic bond and Leu54 inactive site - CH3 with the hydrophobic bond. The distances among pharmacophore respectively are 3,603 A, 6,768 A and 7,345A. It has higher score ( $\Delta \mathrm{G}_{\text {bind: }}-3.4 \mathrm{kcal} / \mathrm{mol}$ ) compared to linalool ( $\Delta \mathrm{G}$ bind: $-3.6 \mathrm{kcal} / \mathrm{mol})$. Virtual tethering of linalool with LDI Aspergillus niger enzyme in amino acid Leu120 and Glu118 had been done. The pharmacophore are- $\mathrm{OH}$ and methyl $\mathrm{C}_{8}$ group. The distances among pharmacophore respectively are 5,835 $\AA, 2,52 \AA$ and 5,32 A. Virtual tethering of LDI Aspergillus niger enzyme with geraniol has a higher score $(\Delta \mathrm{Gbind}$ : $-3.4 \mathrm{kcal} / \mathrm{mol})$ compared to linalool $(\Delta \mathrm{Gbind}:-3.6 \mathrm{kcal} / \mathrm{mol})$. It shows that interaction between linalool and LDI Aspergillus niger enzyme is easier to occur than the interaction between geraniol and LDI Aspergillus niger enzyme, geraniol reaction to linalool that occurs is rearrangement reaction.
\end{abstract}

Key words: Aspergillus niger, Docking molecular, Geraniol, Linalool Dehydratase-Isomerase, Comparative modelling.

\section{INTRODUCTION}

Geraniol is a very valuable aroma chemical and has wide use in fragrances and aroma compound. There are three sources producing this chemical aroma. Palmarosa Oil Cymbopogon martini commonly known as Rosha' or Russa is the main source of geraniol (80 - 95\%) and Jamrosa oil contains $80-89 \%$. Other geraniol sources are Cymbogopon winterianus (Javanese lemongrass oil) containing 40-45\% including citronellol. (Akbar N. Saxena B. K, 2009) Biotransformation of geraniol, nerol and citral by Aspergillus niger has been studied. Main bioconversion product obtained from geraniol and nerol with liquid culture A. Niger are linalool and alpha-terpineol. (Demyttenaere, Del Carmen Herrera and

\footnotetext{
*Corresponding author : Shirly Kumala
}

Email : fskumala@yahoo.com
De Kimpe, 2000) Linalool plays a major role in antiinflammatory activities. (Panin et al., 2002) Antibacterial and antioxidant activities of $\alpha$ terpineol, linalool, eucalyptol and $\alpha$-pinene obtained from essential oil, against pathogenic forming bacteria and decomposers have also been determined. Bacterial activities of this compound are observed in vitro on four Gram-negative and three Gram-positive strains. S. putrefaciens is the most resistant bacteria of all tested components with the value of MIC $2 \%$ or higher, whereas E. coli 0157: H7 is the most sensitive strain among the tested bacteria. Eucalyptol extends lag phase of $S$. typhimurium, E coli )157: H7 and S. aureus at 0.7, $0.6 \%$ and $1 \%$ concentrations respectively. (Zengin and Baysal, 2014)

Recognition of microbial biotransformation as an important manufacturing tool has increased in the chemical and pharmaceutical industries. 
Biotransformation can be clarified as a specific modification of certain compound to different products with structural similarity, by means of biological catalysis including microorganism. Biological catalysis can be described as an enzyme or whole microorganism. (Hegazy et al., 2015)

Aspergillus niger is a famous fungus that has been used for various organic compound biotransformations. A. niger adds hydroxyl, carbonyl and other groups in a certain position or reduces double bonds to produce new valuable compounds. (Parshikov and Sutherland, 2014) Aspergillus niger is a broad aerobic fungus that has been used for various biotransformation. Monoterpene that is commonly used in the pharmaceutical product is containing two terpene molecules; isoprene units and derivatives. ( $\mathrm{Hu}$ et al., 2017)

Biotransformation of $( \pm)$-linalool with submerged Aspergillus niger culture particularly $A$. niger ATCC 9142 produces a mixture of linalool oxide cis and trans-furanoid (15-24\%) and cis- and trans- pyranoid linalool oxide (5-9\%). Biotransformation of $(\mathrm{R})-(-)$ - linalool with the same strains produces almost pure linalool oxide trans-furanoid and trans-pyranoid (ee> 95). This conversion is purely biocatalytic since in acidified water ( $\mathrm{pH}<3.5$ ), almost $50 \%$ linalool is regained unchanged, the remaining is lost by evaporation. (Demyttenaere and Willemen, 1998) This A. niger strain can only convert ( - ) beta-pinene to alphaterpineol (4\%). (Toniazzo et al., 2005)

$A$. niger produces several extracellular enzymes for significant industrial purposes, including amylase, protease, pectinase, lipase and chitin. (Raper KB, 1965) (Shubakov AA, 2002) $A$. niger also degrades cellulose and hemicelluloses (Raper KB, 1965) (Ademark P, Varga A, Medve J, Harjunpää V, Drakenberg T, Tjerneld F, 1998) and results in biodeterioration of lubricant derived from oil, (Yemashova NA, Murygina VP, Zhukov DV, Zakharyantz AA, Gladchenko MA, Appanna V, 2007) polyvinyl chloride and starch plastic/polyethylene. (Shah AA, Hasan F, Hameed A, 2008) The ability of $A$. niger culture to produce acid citrate and vitamin, oxalate, gluconate, fumarate and gallate including biotin, thiamin and riboflavin is widely used in the industry. (Raper KB, 1965) Because of its capability, A. niger has become the most often used fungi as a catalyst for biotransformation of different organic compounds. (Borges KB, Borges WdS, Durán-Patrón R, Pupo MT, Bonato PS, 2009) (Ward OP, Qin WM, Dhanjoon J, Ye J, 2006)

Castellaniella (ex Alcaligenes) defragans strain 65 mineralizes monoterpene without oxygen. Anaerobic soluble cell extract catalyzes geraniol isomerization into linalool and linalool dehydration into myrcene. Linalool dehydratase catalyzes two-reaction in vitro in both directions depending on thermodynamic driving force: water separation from linalool tertiary alcohol to suitable acyclic monoterpene myrcene and isomerization of primary geraniol alyl alcohol in linalool steinaloisomer. (Brodkorb et al., 2010) Linalool dehydratase-isomerase of Castellaniella defragans 65 strain catalyzes in thermodynamic direction of $\beta$-myrcene hydration to linalool and then isomerization to geraniol, initial steps in anaerobic $\beta$-myrcene biodegradation. (Lüddeke and Harder, 2011)

This study aims to investigate geraniol interaction in the active site of Aspergillus niger enzyme with docking molecular; however, LDI crystal structure in Aspergillus niger which is not available is required.

\section{METHODOLOGY}

The main tool for comparative modelling is hardware in the form of ASUS brand laptop. In addition to such a tool, the software is also used: SWISS-MODEL (https://swissmodel.expassy.org). The tool for virtual tethering of geraniol with Aspergillus niger model enzyme is similar to the one that is used for comparative modelling of LDI enzyme. In addition to such a tool, software of OpenBabel version 2.3.2, Discovery Studio version 4.1, and Pyrex version 0.8 are also used.

The material for comparative modelling of this LDI enzyme is an amino acid sequence of Aspergillus niger downloaded from Uniprot site (https://www.uniprot.org/) (amino acid sequence: A0A117DZX7) and complex crystal structure of Linalool Dehydrotase Isomerase enzyme with resolution $2.10 \AA$ downloaded from RSCB site (https://www.rcsb.org/) (crystal structural code: $5 \mathrm{i} 3 \mathrm{t}$ ). Material for virtual tethering of geraniol and LDI Aspergillus niger is Aspergillus niger enzyme structure, the output of above comparative modelling and substrate structure (geraniol). Geraniol structure is downloaded from Pubchem site (https://pubchem.ncbi.nlm.nih.gov) (Code CID-637566)

\section{Comparative Modeling Procedure of LDI Enzyme}

At first, the alignment of the amino acid sequence of Aspergillus niger enzyme to the amino acid sequence of each chain from LDI enzyme crystal structure with Swiss-Model software is conducted automatically. (https://sissmodel. expasy.org/interactive/14Mw28/templates/). 


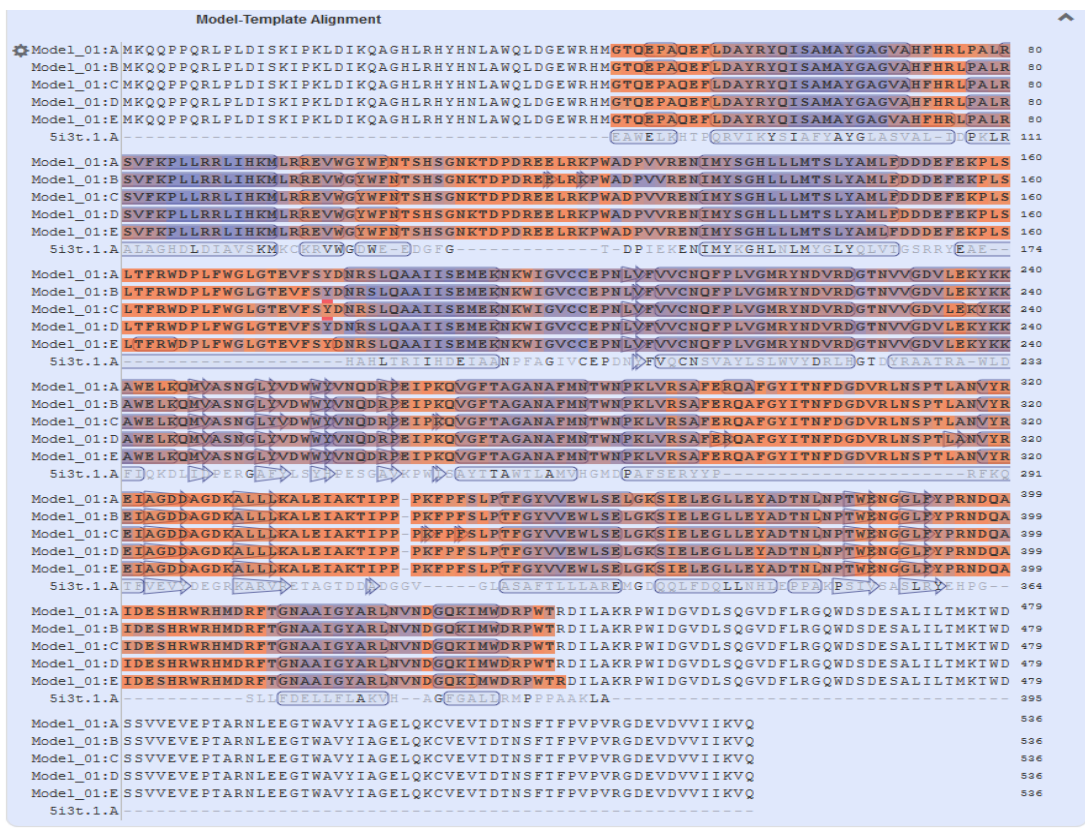

Figure 1. Alignment of Amino Acid sequence of Aspergillus niger Enzyme (Query: > 400 Amino Acid)

Comparative modelling of Aspergillus niger enzyme was then carried out based on the LDI enzyme chain.

\section{Virtual Tethering Procedure of Geraniol and LDI Aspergillus niger}

Geraniol structure and LDI Aspergillus niger enzyme are converted into GDP with Open Babel software and then manually checked with Discovery Studio software.

Virtual tethering of geraniol in Aspergillus niger LDI enzyme is conducted automatically with Pyrex software with the original parameter (default). This virtual tethering produces 8 poses of geraniol tethering with LDI $A$. niger enzyme as well as their tethering energy. The pose of geraniol virtual tethering with LDI $A$. niger enzyme with the most negative tethering energy value is then analyzed by the interaction pattern with LDI enzyme with Discovery studio software. From the analysis, geraniol virtual interaction with LDI Aspergillus niger enzyme is obtained.

\section{RESULT AND DISCUSSION \\ Comparative Modeling of Aspergillus niger Enzyme}

Crystal structure of LDI enzyme is selected as a reference for comparative modelling of Aspergillus niger with respect to Sharper resolution: $\leq 3 \AA$, the most coverage among another closing to 1 and higher similarity.
Crystal structure of LDI enzyme consists of 5 strains (A-E). In Figure 1, it is seen that more than 80\% Aspergillus niger enzyme amino acid can line with acid - the same amino acid (conserved) in LDI enzyme. The high similarity between the amino acid sequence of Aspergillus niger enzyme and amino acid sequence of the LDI enzyme enables to be obtained the relatively accurate Aspergillus niger enzyme model.

The alignment result of Aspergillus niger enzyme model that has been optimized for LDI enzyme is shown in the following (Figure 2).

From Figure 2 it can be seen that the amino acids in the active site, namely Asn 105, Arg 96, Lys 112 and Leu 54 are not in the disallow region. And only $5 \%$ are in the disallow region.

\section{Virtual Tethering of Geraniol and Aspergillus niger Enzyme}

Through analysis of virtual tethering poses, several amino acids of Aspergillus niger enzyme interacting with geraniol are found: Asn105, Lys112, Arg96, Leu54, Arg97. The pharmacophore is $\mathrm{OH}$, methyl $\mathrm{C}_{3}$ and methyl $\mathrm{C}_{8}$ group. The distances among pharmacophore respectively are 3,603 $\AA, 6,768 \AA$ and 7,345 A. Leu54 enzyme holds geraniol tip so that it does not shift far and stable. If geraniol is held steady in enzyme active site (restrained), geraniol has a chance to react. Catalytic amino acids such as Asn105, Lys112, Arg96 assist the reaction process because they 


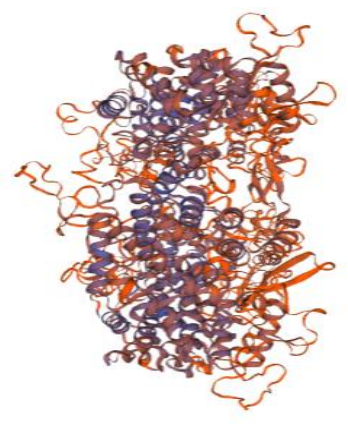

(a)

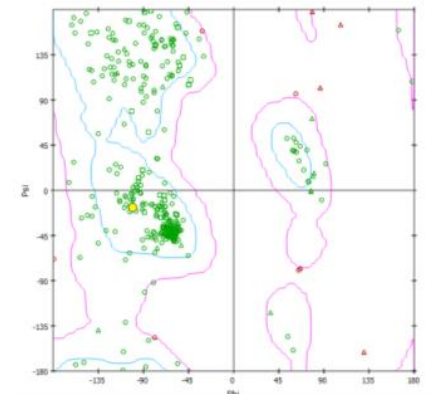

(b)

Figure 2. (a) Model alignment of Aspergillus niger Enzyme (red) against LDI enzyme (green); (b) Ramachandran Map of Aspergillus niger Enzyme Model

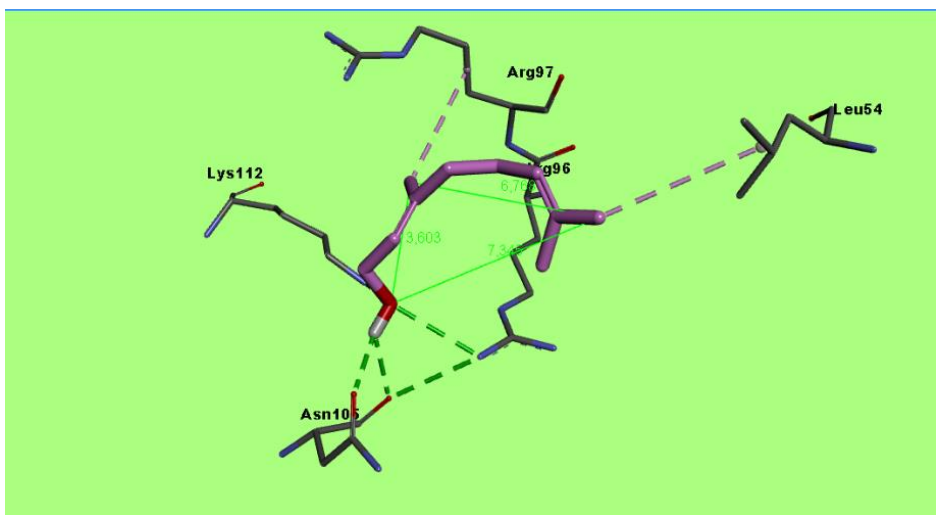

Figure 3. Virtual Attachment Pose of Aspergillus niger Enzyme and Geraniol

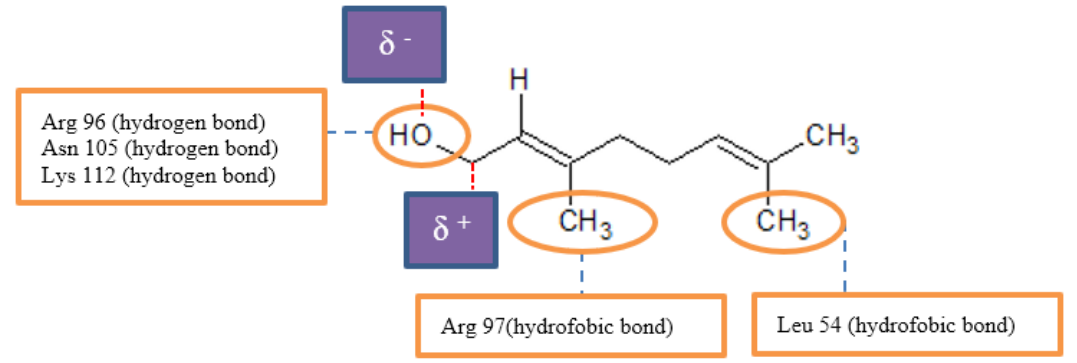

Figure 4. Prediction of geraniol and LDI Aspergillus niger bond

interact with $\mathrm{OH}$ (hydrogen bond) group. The interaction that occurs is an attractive trade-off bond, not a covalent bond. From geraniol virtual tethering in Aspergillus niger enzyme model, geraniol pose with the most negative value of

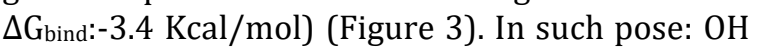
group faces Asn 105, Arg 96, Lys 112 enzymes with hydrogen bond, Methyl faces Leu54 enzyme with the hydrophobic bond. The double bonds face Arg 97 enzyme with a hydrophobic bond (Figure 4). Thus, the geraniol obtained inaccurate virtual tethering pose.
The possibility of the mechanism of geraniol change reaction to linalool is rearrangement reaction. The orientation of such $\mathrm{OH}$ group supports geraniol rearrangement reaction to linalool through the mechanism of rearrangement action. (Figure 5). $\mathrm{OH}$ is the good leaving group. $\mathrm{OH}$ is drawn by 3 amino acids at once so that $\mathrm{OH}$ is easy to escape and then a rearrangement reaction takes place. This is supported by the result of a study reported by Cori et al., (Cori et al., 1986)

In common synthesis, the acid ambience is required as $\mathrm{H}^{+}$donor to protonize $\mathrm{OH}$. $\mathrm{OH}$ captures 


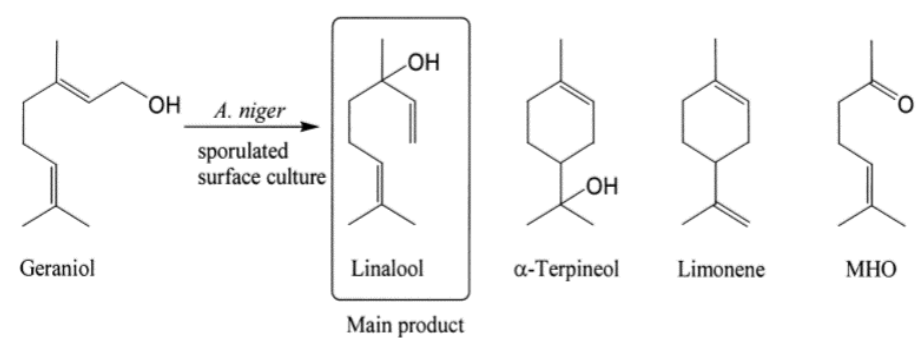

Figure 5. Biotransformation of Geraniol with Aspergillus niger (Demyttenaere et al., 2000)

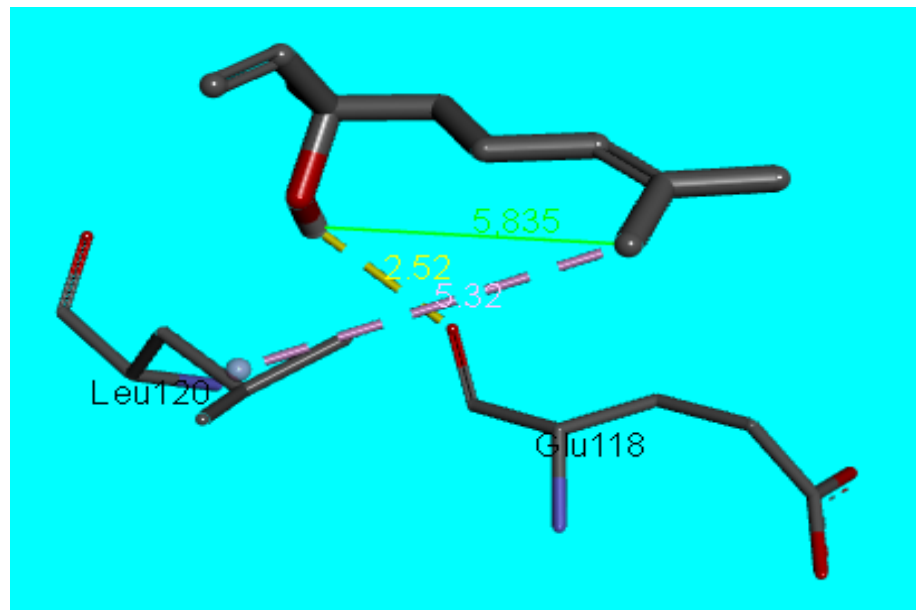

Figure 6. Virtual tethering pose of Aspergillus niger enzyme and Linalool

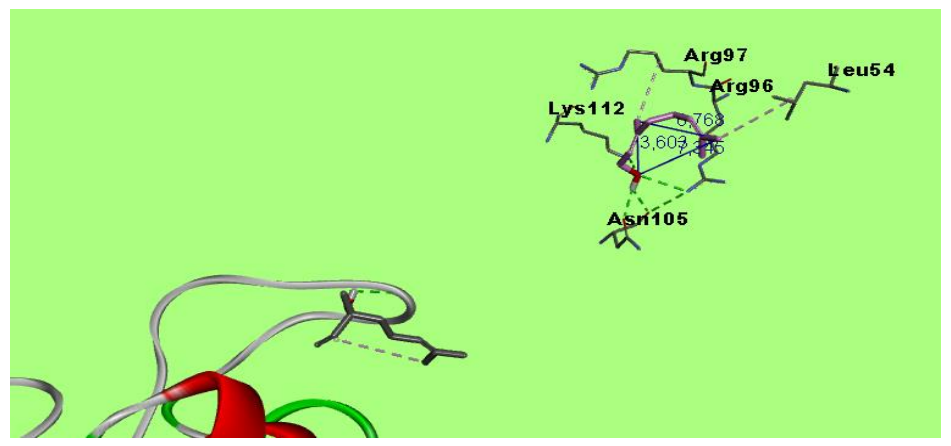

Figure 7. Interaction between Linalool and Geraniol in LDI Aspergillus niger LDI

captures $\mathrm{H}^{+}$tp $\mathrm{OH}_{2}+$. Simultaneously $\mathrm{OH}$ is released, the double bond is displaced, $\mathrm{OH}$ occupies position C in the previous double bond. Takeo et al., (1991) reported the transformation rate from geraniol to linalool depending on $\mathrm{pH}$ and transformation rate is proportional to the concentration of hydrogen (H:) in shochu model solution. (Cori et al., 1986) (Takeo Ohta, Yuzo Morimitsu, Yoshihiro Sameshima, 1991)

Virtual tethering of linalool with LDI Aspergillus niger enzyme in amino acids Leu120 and Glu 118. The pharmacophore is $\mathrm{OH}$ and methyl, $\mathrm{C}_{8}$ group. The distances among pharmacy respectively are $5,835 \AA, 2,52 \AA$ and $5,32 \AA \AA$.

Virtual tethering of LDI Aspergillus niger with linalool has the score of $\Delta \mathrm{G}_{\text {bind: }}-3,6 \mathrm{Kcal} / \mathrm{mol}$ (higher than geraniol: $\Delta \mathrm{G}_{\text {bind: }}-3,4 \mathrm{Kcal} / \mathrm{mol}$ ). This shows that interaction between linalool and LDI Aspergillus niger enzyme is easier than the interaction between geraniol and LDI Aspergillus niger enzyme since it releases higher energy which is similar to what was reported by Brodkorb D et al., (2010). (17) 
Figure 7 above shows that the interaction between geraniol and linalool with LDI Aspergillus niger LDI occurs in different positions.

\section{CONCLUSION}

Virtual tethering of LDI Aspergillus niger enzyme with geraniol has a higher score $\left(\Delta \mathrm{G}_{\text {bind: }}\right.$ $3,4 \mathrm{Kcal} / \mathrm{mol})$ compared to linalool $\left(\Delta \mathrm{G}_{\text {bind: }}-3,6\right.$ $\mathrm{Kcal} / \mathrm{mol}$ ). This shows that interaction between linalool and LDI Aspergillus niger enzyme is easier to occur compared to the interaction between geraniol and LDI Aspergillus niger enzyme since it releases higher energy. The occurring reaction geraniol to linalool is rearrangement reaction.

\section{REFERENCES}

Ademark P, Varga A, Medve J, Harjunpää V, Drakenberg T, Tjerneld F, et al. (1998) 'Softwood hemicellulose-degrading enzymes from Aspergillus niger: purification and properties of a $\bigotimes$-mannanase.', J Biotechnol, 63, pp. 199-210.

Akbar N. Saxena B. K (2009) 'Isolation of Geraniol content from various essential oils', C(1), p. 5890.

Borges KB, Borges WdS, Durán-Patrón R, Pupo MT, Bonato PS, C. I. (2009) 'Stere- oselective biotransformations using fungi as biocatalysts.', Tetrahedron Asym, 20, pp. 385-97.

Brodkorb, D. et al. (2010) 'Linalool DehydrataseIsomerase, a Bifunctional Enzyme in the Anaerobic Degradation of Monoterpenes * 口', 285(40), pp. 30436-30442.

Cori, O. et al. (1986) 'Linalool, Geraniol, and Nerol and Their Derivatives', (15), pp. 1310-1316.

Demyttenaere, J. A. N. C. R. and Willemen, H. M. (1998) 'Biotransformation of linalool to Furanoid and Pyranoid Linalool Oxides by Aspergillus niger', 47(6), pp. 1029-1036.

Demyttenaere, J. C. R., Del Carmen Herrera, M. and De Kimpe, N. (2000) 'Biotransformation of geraniol, nerol and citral by sporulated surface cultures of Aspergillus niger and Penicillium sp.', Phytochemistry, 55(4), pp. 363-373.

Hegazy, M. E. F. et al. (2015) 'Microbial biotransformation as a tool for drug development based on natural products from mevalonic acid pathway: A review', Journal of Advanced Research. Cairo University, 6(1), pp. 17-33.
$\mathrm{Hu}$, S. et al. (2017) 'Research progress on biotransformation of monoterpenes by Aspergillus niger Monocyclic monoterpenes monoterpenes Monocyclic monoterpenes', 12(6).

Lüddeke, F. and Harder, J. (2011) 'Enantiospecifi c $(S)-(+)$-Linalool Formation from $\beta$ Myrcene by Linalool DehydrataseIsomerase', pp. 1-4.

Panin, F. et al. (2002) 'Anti-inflammatory activity of linalool and linalyl acetate constituents of essential oils', pp. 721-726.

Parshikov, I. A. and Sutherland, J. B. (2014) 'The use of Aspergillus niger cultures for biotransformation of terpenoids', Process Biochemistry. Elsevier Ltd.

Raper KB, F. D. (1965) The genus Aspergillus. Baltimore: Williams \& Wilkins;

Shah AA, Hasan F, Hameed A, A. S. (2008) 'Biological degradation of plastics: a comprehensive review.', Biotechnol Adv, 26, pp. 246-65.

Shubakov AA, E. E. (2002) 'Production of polygalacturonases by filamentous fungi Aspergillus niger ACM F-1119 and Penicillium dierckxii ACIM F-152.', Chem Com- put Sim Butlerov Commun, 2, pp. 65-8.

Takeo Ohta, Yuzo Morimitsu, Yoshihiro Sameshima, T. S. and T. O. (1991) "Transformation from Geraniol, Nerol and Their Glucosides into Linalool and Terpineol during Shochu Distillation', Fermentation, Journal O F, 72(5), pp. 347351.

Toniazzo, G. et al. (2005) 'Biotransformation of (Jbeta-pinene by Aspergillus niger ATCC 9642.', Applied biochemistry and biotechnology, 121-124, pp. 837-44. doi: 10.1385/ABAB:123:1-3:0837.

Ward OP, Qin WM, Dhanjoon J, Ye J, S. A. (2006) 'Physiology and biotechnology of Aspergillus.', Adv Appl Microbiol 2006;58:175., 58, pp. 1-75.

Yemashova NA, Murygina VP, Zhukov DV, Zakharyantz AA, Gladchenko MA, Appanna $\mathrm{V}$, et al. (2007) 'Biodeterioration of crude oil and oil derived products: a review.', Rev Environ Sci Biotechnol, 6, pp. 315-37.

Zengin, H. and Baysal, A. H. (2014) 'Antibacterial and Antioxidant Activity of Essential Oil Terpenes against Pathogenic and SpoilageForming Bacteria and Cell StructureActivity Relationships Evaluated by SEM Microscopy', pp. 17773-17798. 